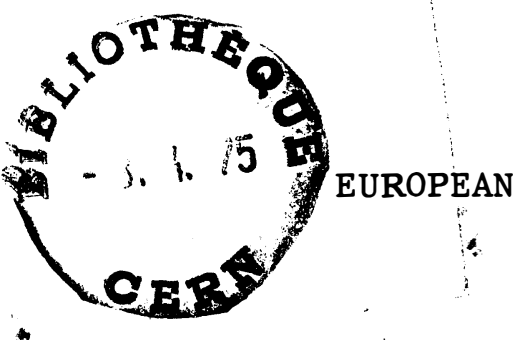

ORGANIZATION FOR NUCLEAR RESEARCH

CERN/ISR-RF-DI-TH-OP/75-15

\title{
EFFECTS OF SPACE CHARGE AND REACTIVE WALL IMPEDANCE
}

ON BUNCHED BEAMS

by

S. Hansen, H.G. Hereward, A. Hofmann, K. Hübner, S. Myers
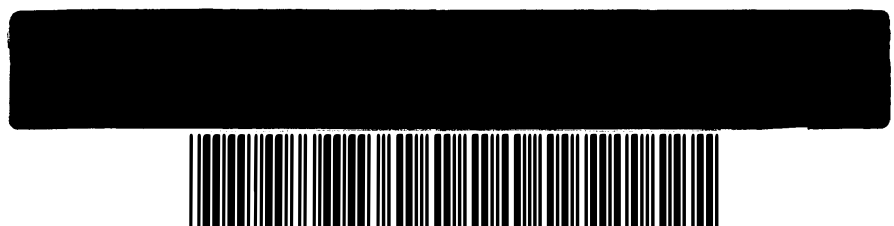

CM-P00064794

To be presented to the 1975 Particle Accelerator Conference, Washington, D.C., March 12-14, 1975 
1. Introduction

2. Measurement of the phase oscillation frequency shift and estimation of the inductive wall impedance

3. Effect of the frequency shift on longitudinal stability

4. Bunch lengthening

5. Reduction of the RF-bucket size

6. Effects of the inductive impedance on beam stacking

References 


\section{EFFECTS OF SPACE CHARGE AND REACTIVE WALL IMPEDANCE}

ON BUNCHED BEAMS

S. Hansen, H.G. Hereward, A. Hofmann, K. Hübner, S. Myers

C E R N

Geneva, Switzerland

Space charge and reactive wall impedance create longitudinal forces inside the bunch which change the incoherent phase oscillation frequency, the bunch length and the size of the RF-bucket. These effects have been investigated with bunched beams in the ISR. By measuring the shift of the quadrupole mode phase oscillation frequency, the strength of the self-forces was determined. The inductive wall is dominant and its impedance (divided by the mode number) was measured to be $|\mathrm{z}| / \mathrm{n} \approx 26 \mathrm{0hms}$. An increase of bunch length with current was measured. It can be explained by the inductive impedance up to a certain current; beyond that an excessive, unexplained bunch lengthening occurs. The reduction of the bucket size affects the stacking process. By correcting for it, an increased density of the stacked beam was achieved.

\section{Introduction}

The longitudinal forces on a beam depend on its surroundings. The space charge forces of a beam in a perfectly conducting chamber have been calculated by Nielsen et al.1). A resistive surrounding can lead to the resistive wall instability, as shown by Neil and Sessler 2). An elegant method to deal with an inductive wall has been developed by Neil and Briggs 3 ); we will use it here. The effect of a general wall impedance has been treated by Sessler and Vaccaro ${ }^{4}$ ).

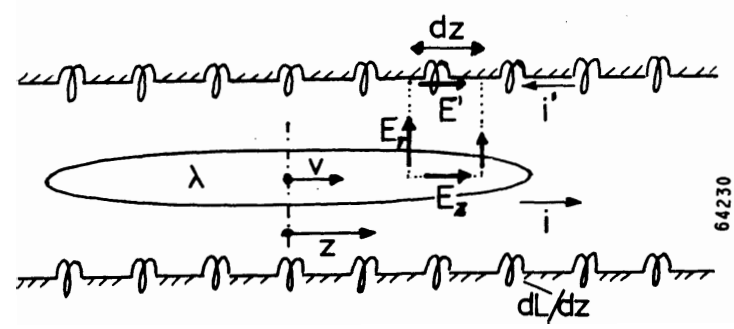

Fig. 1, Fields with inductive wall

We consider a bunch with a charge per unit length e $\lambda$ moving with velocity $v=\beta c$ in a circular pipe as shown in fig. 1. The wall is considered to be perfectly conducting but has a distributed inductance $\mathrm{dL} / \mathrm{dz}$ per unit length. We assume circular symmetry in the transverse directions and a line density $\lambda$ which does not change much over a longitudinal distance of order of the chamber radius. The line charge produces a radial electric field $E_{\mathbf{r}}$ which is proportional to $\lambda 1$ ). The surface charges induced by this field on the chamber produce a wall current $i^{\prime}$ which has the same magnitude but opposite sign as the ac-component of the local beam current $i$. This current $i$ ' gives an electric field $E^{\prime}$ in the distributed wall inductance ${ }^{3}$ )

$$
E^{\prime}=+\frac{d L}{d z} \frac{\partial i^{\prime}}{\partial t}=-\frac{d L}{d z} \frac{\partial i}{\partial t}=+\frac{d L}{d z} \text { e } \beta^{2} c^{2} \frac{\partial \lambda}{\partial z} \text {. }
$$

By calculating the line integral $\overrightarrow{\xi E} \overrightarrow{\mathrm{d}}$ along the dotted path indicated in fig. 1. the longitudinal field $E_{z}$ in the beam is obtained

$$
E_{z}=-e \frac{\partial \lambda}{\partial z}\left[\frac{g_{0}}{4 \pi} \frac{\varepsilon_{0}}{\gamma^{2}}-\frac{d L}{d z} \beta^{2} c^{2}\right],
$$

where $g_{0}$ is the well known coupling coefficient. For a circular beam of radius a in a circular pipe of radius $b$ this coefficient is $g_{0}=1+2 \ln (b / a)$. In the general case, where there is no circular symmetry, the situation is more complicated, but the properties of the wall can always be described by a coupling coefficient go and an inductance per unit length $\mathrm{dL} / \mathrm{dz}$ which is seen by the bem. Integrating the longitudinal field $\mathrm{E}_{\mathbf{z}}(1)$ over the circumference $2 \pi R$ of the machine gives the voltage $U_{z}$ per turn, seen by a particle which is at a distance $z$ from the bunch centre

$$
\mathrm{U}_{z}=-\mathrm{e} \frac{\partial \lambda(z)}{\partial z}\left[\frac{\mathrm{R} \mathrm{g}_{\mathrm{o}}}{2 \varepsilon_{\mathrm{o}} \gamma^{2}}-\mathrm{L} \beta^{2} \mathrm{c}^{2}\right]
$$

where $\mathrm{L}$ is the total inductance per turn.

We assume now bunches with a parabolic line density distribution

$$
\lambda(z)=\frac{6 N}{\ell^{3}}\left[\frac{\ell^{2}}{4}-z^{2}\right], \frac{\partial \lambda}{\partial z}=-\frac{12 N}{\ell^{3}} z
$$

with $\ell=$ full bunch length, $N=$ number of particles per bunch. This parabolic distribution is a very good approximation for the proton bunches in the ISR and produces self-fields which are linear in $z$. Using the total beam current $I=e M N f_{O}$ ( $M=$ number of bunches, $f_{O}=r e v$. frequency) and replacing the total inductance $L$ by its impedance $|z|=\omega L$ divided by the mode number $n=\omega / 2 \pi f_{0}$

$$
\left|\frac{\mathrm{z}}{\mathrm{n}}\right|=2 \pi \mathrm{f}_{\mathrm{O}} \mathrm{L}
$$

gives for the voltage per turn

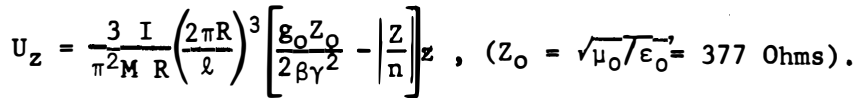

The external RF-voltage

$\mathrm{U}_{\mathrm{RF}}=\mathrm{U}_{\mathrm{O}} \sin \emptyset \sim \mathrm{U}_{\mathrm{O}}\left(\left(\phi-\emptyset_{\mathrm{s}}\right) \cos \emptyset_{\mathrm{s}}+\sin \emptyset_{\mathrm{s}}\right) \approx-\mathrm{U}_{\mathrm{O}} \cos \phi_{\mathrm{s}} \frac{\mathrm{h}}{\mathrm{R}} \mathrm{z}+\mathrm{U}_{\mathrm{O}} \sin \phi_{\mathrm{s}}$

( $h=$ harmonic number, $\emptyset_{S}=$ synchronous phase angle) has to be added to $\mathrm{U}_{\mathbf{z}}$. The total voltage is

$$
\begin{aligned}
U=-U_{\circ} \frac{h}{R} \cos \emptyset_{S}(1 & \left.-\frac{3}{\pi^{2} \frac{I}{h M U}-\cos \emptyset_{s}}\left(-\frac{2 \pi R}{\ell}\right)^{3}\left[\frac{8_{0} Z_{o}}{2 \beta \gamma^{2}}-\left|\frac{z}{n}\right|\right]\right) z \\
& +U_{0} \sin \emptyset_{S} .
\end{aligned}
$$

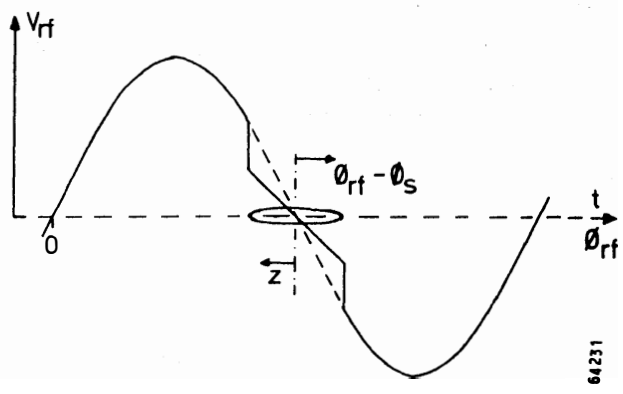

Fig.2. RF-voltage with inductive wall 
The RF-wave form, seen by the beam, is modified by the self-fields as shown in fig. 2 .

Above transition energy, $\cos \emptyset_{\mathrm{s}}$ is negative and the space charge forces increase the effective RF-voltage, the wall inductance decreases it. The opposite is true below transition energy.

The self-forces do not have any effect on the rigid bunch motions because the potential of the self-fields moves with the bunch. However, the internal motion of the particles in the bunch is affected.

There are three main effects of the space charge forces and the wall inductance on bunched beams which are important for the ISR :

a) The incoherent phase oscillation frequency is changed.

b) The bunch length is changed.

c) The size of the RF-bucket is changed.

2. Measurement of the phase oscillation frequency shift. and estimation of the inductive wall impedance.

The incoherent phase oscillation frequency is determined by the slope of the total voltage seen by the beam

$$
f_{s}^{2} \propto\left|\frac{\partial U}{\partial \phi}\right|_{\emptyset=\emptyset_{s}}
$$

Using equation ( 3 ) yields

$$
f_{s}^{2}=f_{s o}^{2}\left(1-\frac{3 I}{\pi^{2} h M U_{o} \cos \phi_{s}}\left(\frac{2 \pi R}{l}\right)^{3}\left[\frac{g o z_{o}}{2 \beta \gamma^{2}}-\left|\frac{z}{n}\right|\right]\right),
$$

where $f_{s o}$ is the frequency in the absence of self-forces. If the effect is small an approximation can be used

$\frac{\Delta f_{S}}{f_{s O}}=\frac{f_{S}-f_{S O}}{f_{S O}} \approx-\frac{3 I}{2 \pi^{2} h M U_{O} \cos \phi_{S}}\left(\frac{2 \pi R}{\ell}\right)^{3}\left[\frac{g_{o} z_{O}}{2 \beta \gamma^{2}}-\left|\frac{z}{n}\right|\right]$.

By measuring the frequency shift $\Delta \mathrm{f}_{S}$ the strength of the self-forces can be determined. A direct measurement of the incoherent frequency is difficult. However we can easily measure the frequencies $f_{\mathrm{Sm}}$ of the coherent higher modes, like the quadrupole mode $(m=2)$, the sextupole mode $(m=3)$ etc. One has to know how much these frequencies are affected. The frequency of the coherent dipole mode does not change $f_{s 1}=f_{s o}$. The coherent higher modes, however, are affected, because they do not represent rigid bunch motions. Since they modulate the self-potential, the effect will be less than for the incoherent oscillations. The magnitude of the frequency shift of the higher modes has been calculated by Hereward and Sфrensen 5) and by Sacherer 6),7).

For the quadrupole mode it is

$$
f_{s 2}-2 f_{s o}=\frac{1}{2} \Delta f_{s} \text {. }
$$

We used the quadrupole mode to determine the strength of the self forces in the ISR

Firstly, the frequency $f_{s} 2$ was measured directly as a function of current. The results are shown in fig. 3. From the slope we can determine the "effective" impedance in the square brackets of (5)

$$
\frac{z_{\text {eff }}}{n}=-\left(\frac{g_{0} z_{o}}{2 \beta \gamma^{2}}-\left|\frac{z}{n}\right|\right) \text {. }
$$

The measurement shows clearly a decrease of the quadrupole mode frequency with increasing current. Since we are above transition energy, this indicates that the inductive impedance is dominant. Similar measurements have been carried out at SPEAR ${ }^{8}$ ).

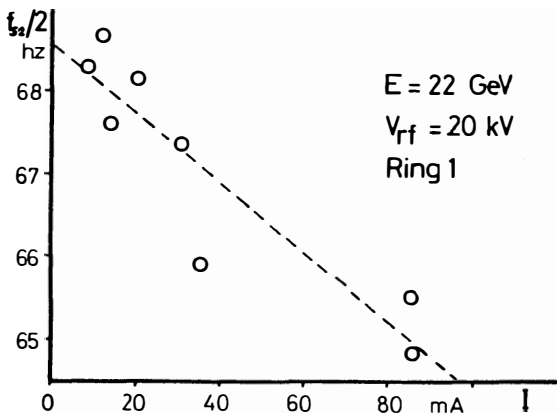

Fig. 3. Synchrotron frequency vs. beam current

Secondly, the frequency of the quadrupole mode was compared directly with that of the coherent dipole mode. The bunches which are injected from the PS have to be matched to the RF-bucket in the ISR. By making this matching not perfect, quadrupole mode oscillations were excited. Furthermore, coherent dipole mode oscillations were obtained by turning the "RF phase-lock" off. 'Due to the self-forces the quadrupole mode frequency is not exactly twice the coherent dipole frequency and an interference between the two modes occurs. The bunches wereobserved on a special "mountain range" display which is shown in fig. 4. The scope was triggered every revolution. Each trace shows a superposition of many sweeps. Depending on the relative phase between the two modes, this superposition looks different. From the period with which this pattern repeats itself, one obtains the difference between the quadrupole mode frequency and twice the coherent dipole frequency

$$
\left|f_{s 2}-2 f_{s 1}\right|=\frac{1}{2}\left|\Delta f_{s}\right| \text {. }
$$

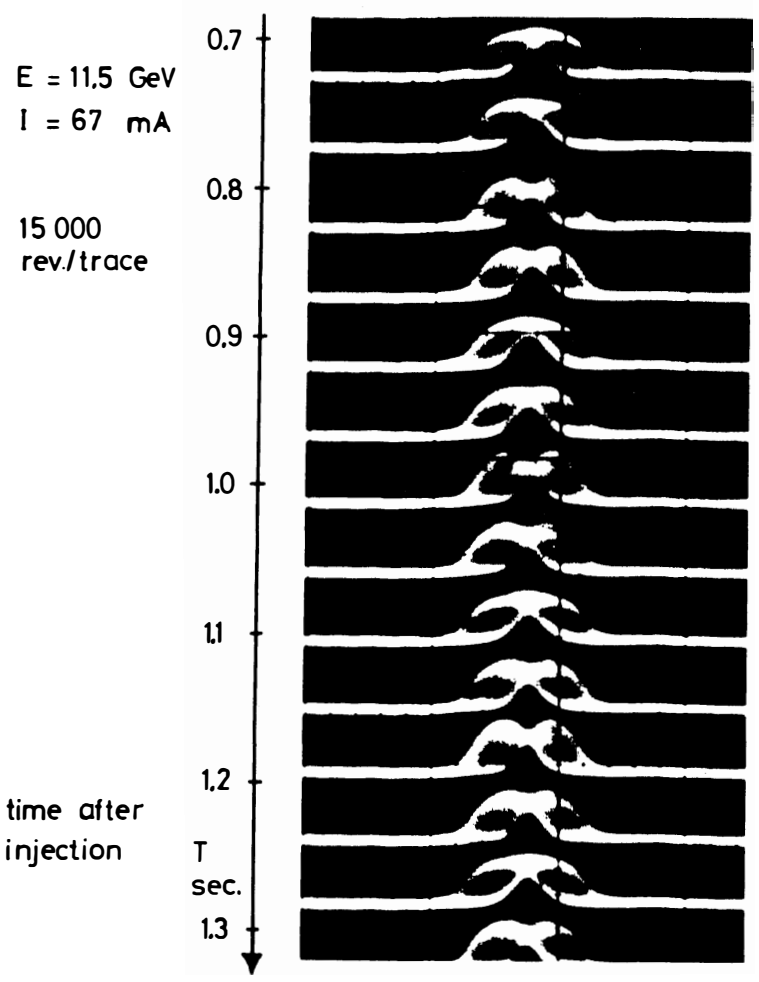

Fig. 4. Interference between dipole and quadrupole mode oscillations 
To exclude other effects, such as coupled bunch mode instabilities, which could also produce frequency shifts, some measurements were done with only 4 instead of 20 bunches. The same frequency shift was observed.

Using equation (4) the "effective" impedance was calculated from the frequency shift measurements. The results are ploted in fig. 5 against $\beta^{-1} \gamma^{-2}$. The value of the "effective" impedance extrapolated to infinite energy $\left(\beta^{-1} \gamma^{-2}=0\right)$ gives the inductive impedance and from the slope in fig. 5 the space charge coupling coefficient go can be estimated

$|\mathrm{z} / \mathrm{n}| \approx 26 \Omega$ or $\mathrm{L} \approx 13 \mu \mathrm{h} ; \mathrm{g}_{0} \approx 5.8$.

From the ISR chamber geometry a value of $80 \approx 4$ is expected. The measured $\mathrm{go}$, which has a large error, is not inconsistent with the expectation.

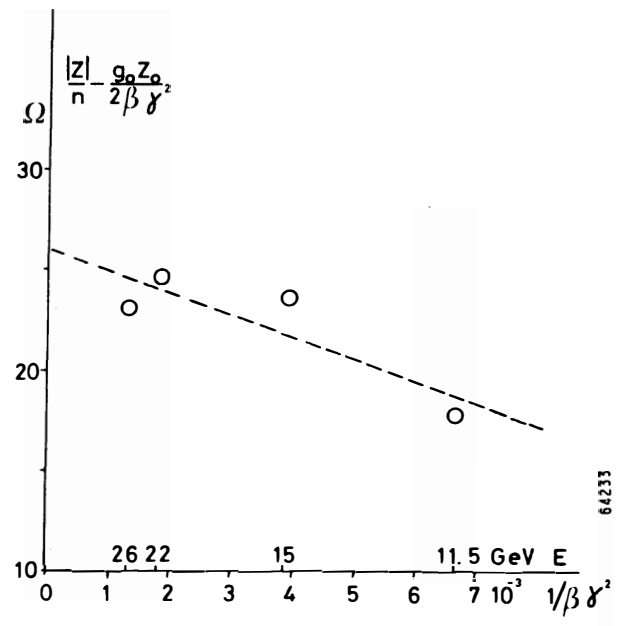

Fig. 5. Effective impedance vs $\beta^{-1} \gamma^{-2}$

At all energies relevant for ISR operation the effect of the inductive wall is dominant. We believe that the relatively large value of the inductive impedance is caused mainly by aperture changes and bellows 9 ). Such elements have resonant frequencies in the Ghz range, and present an inductive impedance at frequencies between 10 and $100 \mathrm{Mhz}$ where the spectrum of the ISR bunches lies. Although it is difficult to estimate the inductive contribution of all the elements seen by the beam, the measured value of $|\mathrm{z}| / \mathrm{n} \approx 26 \Omega$ seems to be reasonable.

\section{Effect of the frequency shift}

The self-forces produce a real shift of the incoherent phase oscillation frequency relative to the coherent frequency, If this shift is comparable or larger than the incoherent frequency spread, there will be no Landau damping and the beam is intrinsically unstable.

In the ISR there is a weak longitudinal instability of bunched beams. Its threshold was measured and found to agree with an expectation which takes the wall inductance into account.

\section{Bunch lengthening}

The inductive impedance reduces the phase focusing above transition energy which results in an increase of the bunch length. This has been known for some timel0) and measurements have been carried out at different machines. The bunch length is related to the incoherent phase oscillation frequency $f_{s}$. This relation is different for protons, where phase space is conserved, and for electrons, where the momentum spread is deter- mined by synchrotron radiation.

For protons the bunch length is related to the phase oscillation frequency by $l / l_{0}=\sqrt{f_{80} / f_{8}}$ where $l_{0}$ is the bunch length in the absence of self-forces. The bunch length is determined by the following equation

$$
\ell^{4}-\frac{24 \pi R^{3} I}{h M U_{0} \cos \emptyset_{s}}\left[\frac{g_{0} Z_{0}}{2 \beta \gamma^{2}}-\left|\frac{\mathrm{Z}}{\mathrm{n}}\right|\right] \ell-\ell_{0}^{4}=0 .
$$

We measured the bunch length in the ISR as a function of current. The bunches were injected from the PS with full intensity. The current was reduced to the desired value in the ISR right after injection by means of a vertical scraper. To check the method, we first measured the bunch length immediately after scraping before the self-forces could establish a new bunch length. Next we measured the bunch length after RFmatching when the final length was established. The results are plotted in fig. 7 and show a clear increase of the bunch length with current. The dashed curve gives the expected bunch lengthening (7) due to the self-forces. The free parameter $l_{0}$ in equation (7) was determined by fitting this equation through the points with I < $100 \mathrm{~mA}$. As fig. 7 shows, the observed bunch lengthening can be explained rather well by the selfforces up to a current of about $140 \mathrm{~mA}$. Beyond this current an unexplained additional increase of the bunch length occurs.

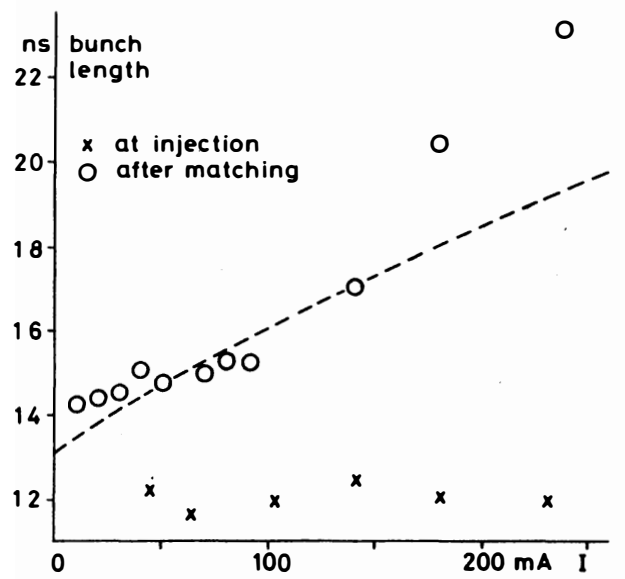

Fig. 6. Bunch length vs. current

5. Reduction of the RF-bucket size

Above transition energy the inductive impedance reduces the size of the bucket and a larger voltage is required to keep a given current bunched than in the absence of self-forces. This is equivalent to the reduction of bucket size due to space charge below transition energy which has been.treated by Nielsen and Sessler 11), by Bigliani 12) and also by Bovet et al 13).

We consider first the case of an injected current of very large phase space density and ask for the maximum proton current which can be kept bunched by a given RF-voltage. This current is reached when the self-fields just about cancel the external $R F-$ focusing everywhere inside the bunch

$$
\mathrm{U}_{\mathrm{RF}}-\mathrm{U}_{\mathrm{O}} \sin \emptyset_{\mathrm{s}}+\mathrm{U}_{\mathrm{z}} \approx 0 \text {. }
$$

Using expression (2) for the self-potential $U_{2}$ this leads to an equation which determines the derivative $e \partial \lambda / \partial z$ of the line charge density. Integrating it twice gives the charge in a bunch and multiplying with 
the number of bunches $M$ and the revolution frequency gives the maximum bunched current

$$
\begin{aligned}
I_{\max }=\frac{\mathrm{MU}_{0}}{2 \pi} \frac{\mathrm{h}^{2} \mid / \mathrm{n}}{} & {\left[\left(\emptyset_{1}-\emptyset_{2}\right) \cos \emptyset_{2}-\sin \emptyset_{1}+\sin \emptyset_{2}\right.} \\
& \left.-\frac{1}{2}\left(\emptyset_{1}-\emptyset_{2}\right)^{2} \sin \emptyset_{\mathrm{s}}\right] .
\end{aligned}
$$

Here $\emptyset_{1}$ and $\emptyset_{2}$ are the maximum and minimum phase angles of the RF-bucket.

The case of an injected current with finite phase space density is more complicated. The maximum current which can be kept bunched by a given voltage depends now also on this density and the line density distribution. We tried to measure this current in the ISR. A beam of $133 \mathrm{~mA}$ and of known phase space density was injected. The RF-voltage was then adiabatically reduced to a final value. All the excessive current was spilled out of the bucket in this process. The current in the final bucket was accelerated dway from the spill out and measured. It is plotted in fig. 7 as a function of the final RF-voltage. The area, the final bucket would have in the absence of self-forces, is shown too on the abscisse. Fig. 7 shows also the current expected for the ISR inductive wall impedance and for the case of no self-forces. Comparison with the measured points indicates some unexpected loss of current, probably the result of a dilution in phase space density. We found before (fig. 6) that for a large RF-voltage (16 kV) such a blow up occurs only for currents larger than $140 \mathrm{~mA}$. Reducing the RF-voltage seems to lower this threshold.

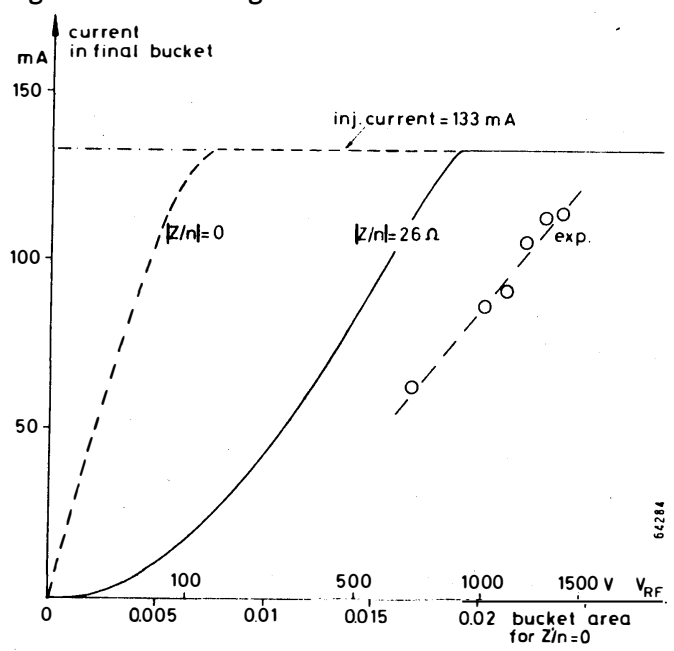

Fig.7. Current in final bucket vs. RF-voltage $(\Gamma=0.5)$

6. Effects of the inductive impedance on beam stacking

Each injected pulse is accelerated in the ISR in three stages. The first acceleration is done in a large bucket, then the bucket area is reduced to $\mathrm{fit}$ tightly around the bunch and the acceleration to the final orbit is done in this small bucket 14). This process is affected by the inductive impedance in several ways.

During reduction of the $R F$-voltage some current is spilled out of the bucket. A second, slow spill-out has been observed during the acceleration in the final bucket. This can be explained qualitatively by the.wall inductance. As long as the bunch is surrounded by the spilled out current, the wall "sees" a smaller derivative $\partial \lambda / \partial z$ of the 1 ine density and the self-forces are reduced. When the bunches are accelerated away from this spill-out the self-forces increase thereby causing a reduction of the bucket size and more current is spilled out. The final condition is reached when all the spill-out is left behind.

The current contained in the final bucket is proportional to the product of the reduced bucket area and the longitudinal density.

In the ISR stacking is performed in momentum space starting at the top. The difference between the momenta at which successive pulses are stacked is calculated from the bucket area. Consequently if self-forces are ignored, the difference will be too large and the resulting density of the stack will be reduced.

During stacking the accelerated pulses may traverse a low density tail of the stack caused e.g. by spill-out. During this traversal the self-forces are reduced and the bucket area increases. Low density beam moves into the growing bucket and may be deposited on the final orbit. The resulting stack density is reduced by this process.

Stacking tests have been performed where the voltage reduction and final acceleration took place within a medium density current stacked before. The later pulses consequently suffer less dilution and build up some high density stack. (The medium density part could be scraped away if it is not anyway driven out of the aperture). Using this technique the maximum stack density was increased by about $40 \%$. At $26 \mathrm{GeV}$ a density of over $0.8 \mathrm{~A} / \mathrm{mm}$ (1560 A/ $/ \Delta$ ) was achieved. This is still smaller than the maximum attainable density but sufficient to produce more than $48 \mathrm{~A}$ of circulating current in the ISR, a value already significantly higher than the current limitation set by other phenomena.

\section{References}

1) C.E. Nielsen, A.M. Sessler, K.R. Symon, Proc. of the Int. Conf. on High Energy Accel. and Instr., CERN (1959) 239.

2) V.K. Neil, A.M. Sessler, Rev. Sci. Instr., 36 (1965) 429 .

3) V.K. Neil, R.J. Briggs, Plasma Physics, 9 (1967) 631 .

4) A.M. Sessler, V. Vaccaro, CERN report 67-2 (1967).

5) H.G. Hereward, A. Sфrensen, Private communication (1970).

6) F. Sacherer, CERN Int. Rep./SI-BR/72-5 (1972).

7) F. Sacherer, Proc. 1971 Particle Accel. Conf., Chicago; IEEE Trans. on Nucl. Sci. 18. (1971) 1105.

8) M.A. Allen et al., Proc. 9th Int. Conf. on High Energy Accel., Stanford (1974) 352.

9) E. Keil, B. Zotter, Particle Accel. 3 (1972) 11.

10) K.W. Robinson, CEAL-TM-183 (1969).

11) C.E. Nielsen, A.M. Sessler, Rev. Sci. Instr. 30. (1959) 8 .

12) U. Bigliani, CERN Int. Rep./SI-EL/68-2 (1968).

13) C. Bovet et al., CERN Int. Rep./MPS-SI-DL/70-4 (1970) 32 .

14) S. Hansen, M.J. de Jonge, Proc. 8th Int. Conf. on High Energy Accel., CERN (1971) 505. 